\title{
A pretreatment method of Volterra the external boundary value problem of integral differential equations
}

\author{
Xiaojuan Chen* \\ School of Road, Bridge and Architecture, Chongqing Vocational College of Transportation, \\ Chongqing, 402247 \\ China \\ Xiaoxiao Ma \\ School of Transportation, Chongqing Vocational College of Transportation, \\ Chongqing, 402247 \\ China
}

Received: January 23, 2021. Revised: August 18, 2021. Accepted: August 29, 2021. Published: August 31, 2021.

\begin{abstract}
In the process of traditional methods, the error rate of external boundary value problem is always at a high level, which seriously affects the subsequent calculation and cannot meet the requirements of current Volterra products. To solve this problem, Volterra's preprocessing method for the external boundary value problem of Integro differential equations is studied in this paper. The Sinc function is used to deal with the external value problem of Volterra Integro differential equation, which reduces the error of the external value problem and reduces the error of the external value problem. In order to prove the existence of the solution of the differential equation, when the existence of the solution can be proved, the differential equation is transformed into a Volterra integral equation, the Taylor expansion equation is used, the symplectic function is used to deal with the external value problem of homogeneous boundary conditions, and the uniform effective numerical solution of the external value problem of the equation is obtained by homogeneous transformation according to the non-homogeneous boundary conditions.
\end{abstract}

Keywords-Boundary value problem, differential equation, Taylor expansion, Volterra integral.

\section{INTRODUCTION}

$\mathrm{D}$ IFFERENTIAL equations are widely used in all sciences, including natural and social sciences. Like differential equations, integral equations are important mathematical tools for describing practical problems, and they have their own advantages in dealing with different problems. For example, the former is usually more convenient to deal with domain problems, while the latter shows its advantages when discussing source problems [1] [2]. For the same problem, when the differential equation is described, because the numerical integration is involved in the process of finding the approximate solution, it often causes a large relative error; But if the integral equation is used to describe, because the relative error caused by the numerical integration is small, although the calculation is large, it is often easy to more ideal result because of the small cumulative error [3]. The differential equation on the domain is transformed into the integral equation on the boundary. Due to the reduction of dimension, the amount of calculation is reduced, so it has been widely used.

The application of integral equations is very extensive, specifically, including many aspects such as aerodynamics, biomechanics, thermodynamics, quantum theory, game theory and automatic control theory [4]. The theory of existence, uniqueness and stability of the solution of integral equation, through the long and unremitting efforts of scholars, the theory is becoming more and more mature, and the results are becoming more and more abundant. Among them, the more classical theory is the Volterra theorem. Although the theory about the existence of the solution of the integral equation is perfect, in practical application, except for the more special cases, the analytical form of the solution of the integral equation is difficult to find, so it is necessary to resort to or approximate the method or numerical method [5]. Numerical or approximate methods have always been paid close attention by Chinese scholars at home and abroad, various methods emerge in endlessly, the accuracy is higher and higher, and the operation is 
more and more simple.

For the treatment of the outer value problem of the Volterra integral differential equation, the common treatment methods abroad are. Brunner. $\mathrm{H}$ iterative configuration method of Volterra integral equation is given. Marchuk. G.I. Shaidurov. V.V. approximate solution of the Volterra integral equation is obtained by using the Nystrom method [6]. Although the above methods are very accurate, they rely more on advanced computers. Compared with numerical solutions, approximate solutions allow errors in a certain range, so the approximate solution method is still adopted by many scholars, and there are iterative methods, spectral methods and so on in the traditional approximate solution. these methods are difficult to match the exact solution for the case of polynomials [7].

The external value problem of Integro differential equations is a practical and active branch in the field of differential equations. The theory of the existence and uniqueness of solutions of external value problems has been very mature. In recent years, scholars have mainly studied its solutions on this problem, especially numerical solutions and approximate solutions [8]. Among these solutions, the most important one is the preprocessing of the external boundary value problem. The traditional preprocessing of the external boundary value problem of Volterra Integro differential equations mostly uses the linear multistep method or spline function method to achieve the purpose of preprocessing, but in the process of processing, the error ratio of the external initial value problem is always at a high level, which seriously affects the subsequent calculation and cannot meet the current Volterra product. It is necessary to deal with the external boundary value problems of partial differential equations. To solve this problem, the Volterra study of the preprocessing method for the external boundary value problem of Integro differential equations is proposed in this paper. Sinc function is used to deal with the external value problem of Volterra Integro differential equation to reduce the error of the external initial value problem. Firstly, the external boundary value problem of Volterra Integro differential equation is analyzed, mainly including the external boundary value problem of oltera Integro differential equation, the transformation of outbound value problem in differential equation, Taylor expansion, the treatment of external value problem of homogeneous boundary conditions and the treatment of external value problem of non-homogeneous boundary conditions. Experiments are carried out to prove the feasibility of the design method.

\section{Solution Of External Boundary Value Problems FOR VOLTERRA INTEGRO DIFFERENTIAL EQUATIONS}

\section{A. Transformation of Outbound Value Problems in Differential Equations}

The problem of the outer value of differential equations often appears in the field of natural science and engineering technology, and more requirements in practical engineering to obtain numerical solutions or approximate solutions. Therefore, the differential equation is transformed into the Volterra integral equation with taking the unknown function and its derivative as the unknown quantity to solve the outer value of the system of Volterra integral differential equations [9].

Taking the second-order linear ordinary differential equation as an example, it is transformed into Volterra integral equation. Before using the third-party software to verify the conclusion of the solution, the second-order linear ordinary differential equation of the external value problem exists in the appropriate space, so as to prove that the second-order linear ordinary differential equation of the preprocessed external value problem has certain significance. The verification results are shown in Fig. 1.

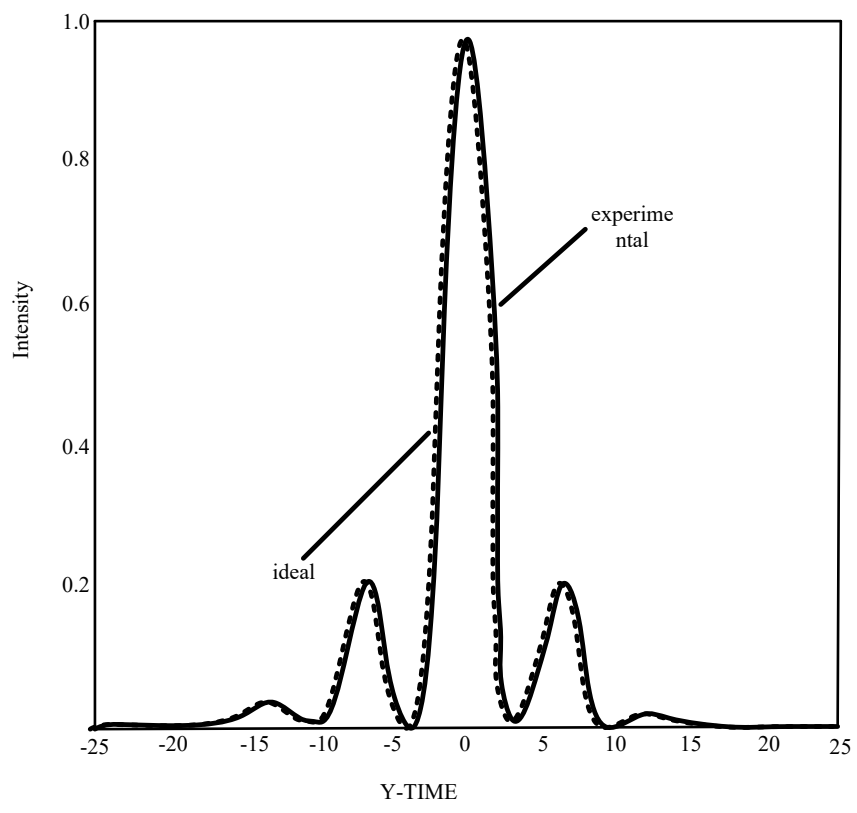

Fig. 1 validation results of the solution of the outer value problem of the second order linear ordinary differential equation in proper space

It can be clearly seen from the figure that the ideal curve is basically consistent with the experimental curve, which proves the existence conclusion of the solution of the outer value problem of the second order linear ordinary differential equation in the proper space [10]. According to the characteristics of the second order linear ordinary differential equation, there are two outer value problems, as shown in Fig. 2. 


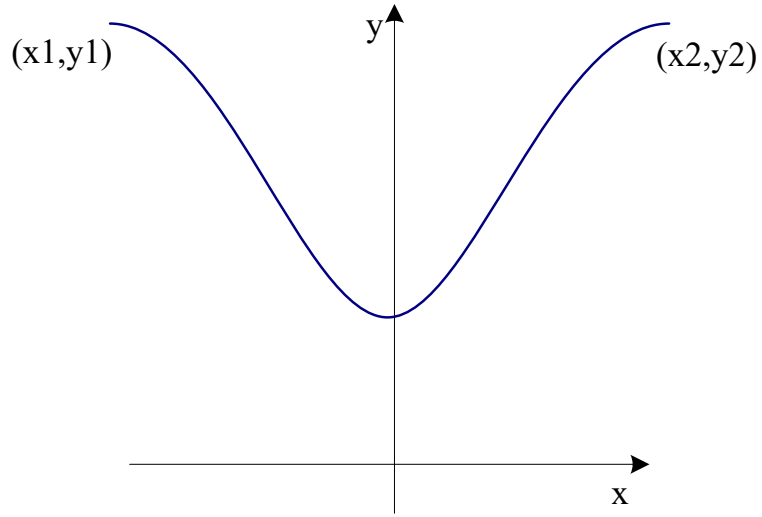

Fig. 2 exterior boundary of second-order linear ordinary differential equations

The second order linear ordinary differential equations are as follows:

$a^{\prime \prime}(x)+b(x) a^{\prime}(x)+c(x) a(x)=f(x)$

where variable coefficients $b(x)$ and $c(x)$ and nonhomogeneous term $f(x)$ are known continuous functions on interval $q$, and $b(x) \in Q(q)$, the initial value problem is studied:

$a\left(x_{0}\right)=\beta_{0}$

$a^{\prime}\left(x_{0}\right)=\beta_{1}$

In the formula $x_{0} \in q$, the $\beta_{0}$ and $\beta_{1}$ are given constants, the $a(x)$ is the function sought and the equation is assumed to have a unique solution [11]. As a result of $b(x) \in Q(q)$, after constant deformation and partial integration, it can be obtained:

$\int_{x_{0}}^{x}(x-t)^{n} b(t) d t=n ! b(x)-b\left(x_{0}\right)\left(x-x_{0}\right)$

where $n$ denotes the order and $t$ the variable. To obtain the integral from $x_{0}$ to $x$ on both sides of (1), value condition and the partial integral.

$$
\begin{aligned}
& a^{\prime}(x)-\beta_{1}+b(x) a(x)-b\left(x_{0}\right) a\left(x_{0}\right)+\int_{x_{0}}^{x}[c(t)-b(t)] a(t) d t \\
& =f_{(q)}(x)
\end{aligned}
$$

The integral from $x_{0}$ to $x$ on both sides of (5) is obtained.

$a(x)+\int_{x_{0}}^{x} V(x, t) a(t) d t=s(x)$

Among them:

$V(x, t)=b(t)+(x-t)\left[c(t)-b^{q}(t)\right]$

$s(x)=f_{(2)}(x)+\beta_{0}+\left\lfloor\beta_{0} b\left(x_{0}\right)+\beta_{1}\right\rfloor\left(x-x_{0}\right)$

So far, the second order linear ordinary differential equation is transformed into the standard Volterra integral equation, formula (6) is the Volterra integral equation, and then it is expanded by Taylor.

\section{B. Taylor expanded Equations}

For convenience, a second-order Taylor expansion is adopted
[12]. i.e.

$$
a(t) \approx a(t)-a^{\prime}(x)(x-t)+\frac{1}{2} a^{\prime \prime}(x)(t-x)^{2}
$$

By substituting (9) into (6) and combining (4), we can get:

$s_{00}^{(V)}(x) a(x)+s_{01}^{(V)}(x) a^{\prime}(x)+s_{02}^{(V)}(x) a^{\prime \prime}(x)=s(x)$

where:

$s_{00}^{(V)}(x)=1+b\left(x_{0}\right)\left(x-x_{0}\right)+c_{(2)}(x)$

$s_{01}^{(V)}(x)=-\left[b\left(x_{0}\right)\left(x-x_{0}\right)^{2}+2 ! c_{(2)}(x)-b_{(2)}(x)\right]$

$s_{02}^{(V)}(x)=\frac{1}{2 !}\left[b\left(x_{0}\right)\left(x-x_{0}\right)^{3}+3 ! c_{(4)}(x)-2 \times 2 ! b_{(3)}(x)\right]$

Formula (5) and formula (1) are obtained twice for the derivative of (6), and formula (9) is substituted into (5).

$s_{10}^{(V)}(x) a(x)+s_{11}^{(V)}(x) a^{\prime}(x)+s_{12}^{(V)}(x) a^{\prime \prime}(x)=s^{\prime}(x)$

where:

$s_{10}^{(V)}(x)=b\left(x_{0}\right)+c_{(1)}(x)$

$s_{11}^{(V)}(x)=1-c_{(2)}(x)-b\left(x_{0}\right)\left(x-x_{0}\right)+b_{(1)}(x)$

$s_{12}^{(V)}(x)=c_{(3)}(x)+\frac{1}{2} b\left(x_{0}\right)\left(x-x_{0}\right)^{2}-b_{(2)}(x)$

Thus formula (1), formula (10) and formula (14) constitute a system of linear equations about $f(x), s(x)$ and $s^{\prime}(x)$. Then we deal with the problem of the outer value of homogeneous and nonhomogeneous boundary conditions respectively.

\section{Treatment of the Outside Value Problem of} Homogeneous Boundary Condition

Sinc function is used to deal with the problem of the outer value of homogeneous boundary conditions. Sinc functions are defined $W$ the real axis as follows:

$\sin c(w)=\frac{\sin (\pi w)}{\pi w}$

Sinc basis function is defined by (19):

$S(\omega, v)(x)=\sin c\left(\frac{x-\omega v}{v}\right)$

If the function $f(x)$ are all defined on the real axis as Sinc function, then for $v>0$, when the following series:

$$
c(f, v)(x)=\sum_{t=-\infty}^{\infty} f(t v) S(t, v)(x)
$$

When converging, it is also called the multinomial expansion of $f(x)$ [13]. In the actual calculation, it is necessary to use the finite term instead of the infinite term in the above formula, for example, let $t=-n, \ldots, n$, where $n$ represents the number of Sinc lattice points, which are defined in an infinite strip region $Q_{s}$ of the attached plane, as shown in Fig. 3 . 


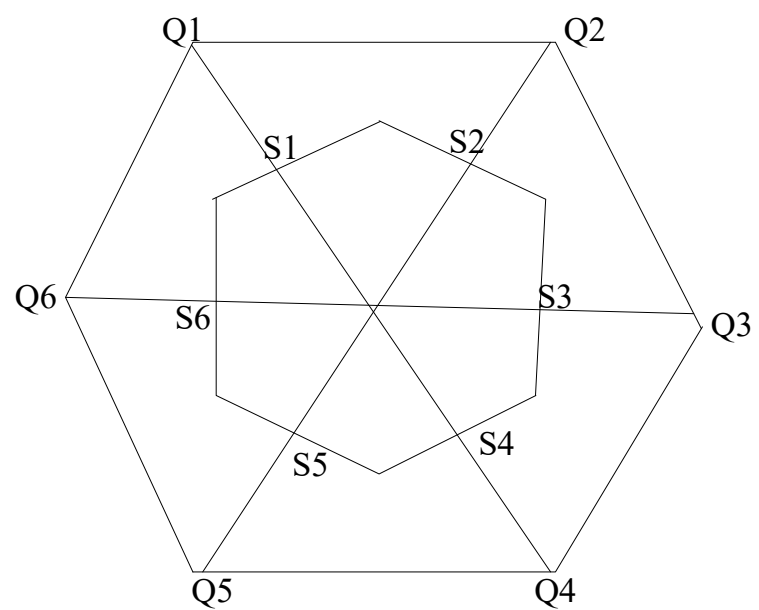

Fig. 3 partition of lattice points in band area

An infinite band region $Q_{s}$ defined as follows:

$Q_{s}=\left\{p=f+t v:|v|<l \leq \frac{\pi}{2}\right\}$

The $p$ in the formula represents the points in the infinite band-shaped region, $l$ the distance between the points in the region. In order to establish an approximation on interval $(\alpha, u)$, consider the following conformal mapping:

$\phi(\chi)=\ln \left(\frac{\chi-\alpha}{u-\chi}\right)$

The eye region $Q_{z}$ is mapped to the infinite belt region $Q_{s}$, and the definition of eye region $Q_{z}$ is as follows:

$Q_{z}=\left\{p \in c:\left|\arg \left(\frac{\chi-\alpha}{u-\chi}\right)\right|<l \leq \frac{\pi}{2}\right\}$

Basis functions in the $\forall p \in Q_{z}$, Sinc method on the interval $(\alpha, u)$ are given by the following compound functions.

$S(t, v) \circ \phi(\chi)=\operatorname{Sinc}\left(\frac{\phi(\chi)-t v}{v}\right)$

Here the function $\chi$ is:

$\chi=\phi^{-1}(p)=\frac{\alpha+u e^{p}}{1+e^{p}}$

Formula (25) is the inverse mapping of $p=\phi(\chi) \cdot \phi^{-1}$ domain on the real axis is:

$\Gamma=\left\{\phi^{-1}(f) \in Q_{z}:-\infty<f<\infty\right\}$

Since Sinc lattice points should be real numbers, $x_{t}$ is used to represent the Sinc lattice $p_{t} \in(\alpha, u)$ on the $Q_{z}$. For equal nodes on a real axis $(t v)_{t=-\infty}^{\infty}$, the corresponding images for these nodes are:

$x_{t}=\phi^{-1}(t v)=\frac{e+u e^{p}}{1+e^{p}}$

where $t=0, \pm 1, \pm 2, \ldots$, the value of the $n$ derivative of the $f(x)$ at the node $x_{t}$ can be approximated by a finite term:

$f^{(n)}\left(x_{t}\right) \cong v^{-n} \sum_{j=-n}^{n} \kappa_{j t}^{(n)} f\left(x_{t}\right)$

Among them:

$\kappa_{j t}^{(n)}=\left.t^{n} \frac{l^{n}}{l \phi^{n}}[S(j, v) \circ \phi(x)]\right|_{x=x_{t}}$

Special items:

$\kappa_{j t}^{(0)}=\left.[S(j, v) \circ \phi(x)]\right|_{x=x_{t}}=\left\{\begin{array}{l}1, j=t \\ 0, j \neq t\end{array}\right.$

$\kappa_{j t}^{(1)}=\left.t \frac{l}{l \phi}[S(j, v) \circ \phi(x)]\right|_{x=x_{t}}=\left\{\begin{array}{l}0, j=t \\ \frac{(-1)^{t-j}}{t-j}, j \neq t\end{array}\right.$

$\kappa_{j t}^{(2)}=\left.t^{2} \frac{l^{2}}{l \phi^{2}}[S(j, v) \circ \phi(x)]\right|_{x=x_{t}}=\left\{\begin{array}{l}\frac{-\pi^{2}}{3}, j=t \\ \frac{-2(-1)^{t-j}}{(t-j)^{2}}, i \neq j\end{array}\right.$

The Volterra system of integral differential equations for homogeneous boundary conditions is studied. it is assumed that the $g(x)$ is an accurate solution to the problem of outer value $s^{\prime}(x)$ the system of integral differential equations. Considering that the series in the polynomial contains infinite terms [14]. A function defined on $[\alpha, u], g(x)$ approximated by the following finite term:

$g(x)=\sum_{j=n}^{n} g_{j} S(j, v) \circ \phi(\chi)$

In the formula, used in the above formula represents matrix multiplier, i.e. matrix point multiplication [15]. $m(x)=s(x, g(x))$. Then there are:

$g^{\prime \prime}(x)+b(x) g^{\prime}(x)+c g(x)+\int_{\alpha}^{x} s_{1}(x, t) m_{1}(t, g(t)) d t+\int_{\alpha}^{x} s_{2}(x, t) m_{2}(t, g(t)) d t$ $=m(x)$

By using (28),

$\left\{\begin{array}{l}g^{\prime}(x) \cong \sum_{j=n}^{n} g_{j} \frac{d}{d x}[S(j, v) \circ \phi(x)] \\ g^{\prime \prime}(x) \cong \sum_{j=n}^{n} g_{j} \frac{d^{2}}{d x^{2}}[S(j, v) \circ \phi(x)]\end{array}\right.$

Of note are:

$\frac{d}{d x}[S(j, v) \circ \phi(x)]=\phi^{\prime}(x) \frac{d}{d \phi}[S(j, v) \circ \phi(x)]$

$\left\{\frac{d^{2}}{d x^{2}}[S(j, v) \circ \phi(x)]\right.$

$=\phi^{\prime \prime}(x) \frac{d}{d \phi}[S(j, v) \circ \phi(x)]+\left(\phi^{\prime}(x)\right)^{2} \frac{d^{2}}{d \phi^{2}}[S(j, v) \circ \phi(x)]$

Put $x=x_{j}, t=-n, \ldots, n$ into formula, $x_{j}$ is the lattice point of Sinc, put (36) into (35), we can get: 


$$
\left\{\begin{array}{l}
g^{\prime}(x) \cong \sum_{j=n}^{n} g_{j} \phi_{t}^{\prime} v^{-1} \kappa_{j t}^{(1)} \\
g^{\prime \prime}(x) \cong \sum_{j=n}^{n} g_{j}\left(\phi^{\prime \prime}{ }_{t} v^{-1} \kappa_{j t}^{(1)}+\left(\phi^{\prime}{ }_{t}\right)^{2} v^{-1} \kappa_{j t}^{(2)}\right)
\end{array}\right.
$$

Among $\phi_{t}^{\prime}=\phi^{\prime}\left(x_{j}\right)$ and $\phi^{\prime \prime}{ }_{t}=\phi^{\prime \prime}\left(x_{j}\right)$. Under these circumstances, assuming that all the Sinc lattice points used in the above calculation process belong to the infinite band-shaped region, by using (27) and (28), and at the same time making $x=x_{t}$, we can obtain from it:

$\left\{\begin{array}{l}\int_{\alpha}^{x_{t}} s_{1}(x, t) m_{1}(t, g(t)) d t=v \sum_{j=n}^{n} \frac{s_{1}}{\phi^{\prime}{ }_{t}} g_{j} \kappa_{j t}^{(1)} m_{1}(t, g) \\ \int_{\alpha}^{x_{t}} s_{2}(x, t) m_{2}(t, g(t)) d t=v \sum_{j=n}^{n} \frac{s_{2}}{\phi^{\prime}{ }_{t}} g_{j} \kappa_{j t}^{(2)} m_{2}(t, g)\end{array}\right.$

By substituting it into (34), as in the case mentioned above, $x=x_{t}, t=-n, \ldots, n$ can obtain:

$\sum_{j=-n}^{n}\left(\phi^{\prime \prime}{ }_{t} \frac{\kappa_{j t}^{(1)}}{v}+\left(\phi_{t}^{\prime}\right)^{2} \frac{\kappa_{j t}^{(2)}}{v^{2}}\right) g_{j}+b_{t} \sum_{j=-n}^{n} \phi_{t}^{\prime} \frac{\kappa_{j t}^{(1)}}{v} g_{j}+c_{t} g_{j}+$

$v b_{t} \sum_{j=-n}^{n} \frac{s_{1}}{\phi_{t}^{\prime}} \kappa_{j t}^{(-1)} m_{1}(t, g)+\sum_{j=-n}^{n} \frac{s_{2}}{\phi_{t}^{\prime}} m_{2}(t, g)=m_{t}$

Among them:

$m_{j}=g\left(x_{j}, s_{t}\right), t=-n, \ldots, n$

According to (40), formula (39) can be sorted out:

$\frac{1}{v} \sum_{j=-n}^{n}\left(\phi^{\prime \prime}{ }_{t}+b_{t} \phi_{t}^{\prime}\right) \kappa_{j t}^{(1)} g_{j}+\frac{1}{v^{2}} \sum_{j=-n}^{n}\left(\phi_{t}^{\prime}\right)^{2} \kappa_{j t}^{(2)} g_{j}+c_{t} g_{j}$

$+b_{t} \sum_{j=-n}^{n} \frac{s_{1}}{\phi^{\prime}{ }_{t}} \kappa_{j t}^{(-1)} m_{1}(t, g)+\sum_{j=-n}^{n} \frac{s_{2}}{\phi^{\prime}{ }_{t}} m_{2}(t, g)=m_{t}$

So far, the external value problem of Volterra Integro differential equation has been transformed into the problem of solving Volterra Integro differential equation. Next, its external value problem is written as a matrix:

$$
\left\{\begin{array}{l}
K^{\varepsilon}=\left[\kappa_{j t}^{(\varepsilon)}\right], \varepsilon=-1,1,2 \\
d(b)=\operatorname{diag}\left(b\left(x_{-n}\right), \ldots, b\left(x_{n}\right)\right) \\
d(c)=\operatorname{diag}\left(c\left(x_{-n}\right), \ldots, c\left(x_{n}\right)\right) \\
d\left(\phi^{n}\right)=\operatorname{diag}\left(\phi\left(x_{-n}\right), \ldots, \phi\left(x_{n}\right)\right) \\
d\left(\phi^{\prime}\right)=\operatorname{diag}\left(\phi^{\prime}\left(x_{-n}\right), \ldots, \phi^{\prime}\left(x_{n}\right)\right) \\
d\left(1 / \phi^{\prime}\right)=\operatorname{diag}\left(1 / \phi^{\prime}\left(x_{-n}\right), \ldots, 1 / \phi^{\prime}\left(x_{n}\right)\right) \\
m_{1}\left(t_{j}, g_{j}\right)=M_{1}\left(t_{1 j}\right) \\
m_{2}\left(t_{j}, g_{j}\right)=M_{2}\left(t_{2 j}\right)
\end{array}\right.
$$

They include: $\left\{\begin{array}{l}\gamma=\frac{1}{v}\left[d\left(\phi^{n}\right)+d(b) d\left(\phi^{\prime}\right)\right] K^{1}+\frac{1}{v^{2}} d\left(\phi^{n}\right)^{2} K^{2}+d(c) \\ G=\left[g\left(x_{-n}\right), g\left(x_{-n+1}\right), \ldots, g\left(x_{n-1}\right), g\left(x_{n}\right)\right]^{T} \\ M=\left[m\left(x_{-n}\right), m\left(x_{-n+1}\right), \ldots, m\left(x_{n-1}\right), m\left(x_{n}\right)\right]^{T}\end{array}\right.$

Formula (41) is simplified as a matrix according to formulas (42) and (43). At this time Volterra the system of integral and differential equations contains $2 n+1$ equations and $2 n+1$ unknown quantities $\left\{g_{j}\right\}_{j=-n}^{n}$. At this time, we can get all the unknown quantities by solving this system of equations, and then we can use $g(x)=\sum_{j=n}^{n} g_{j} S(j, v) \circ \phi(x)$, to obtain the numerical solution of the problem of the outer value of the equation.

The solvability of the external boundary value problem of Volterra Integro differential equation is first expressed as:

$\left\{\begin{array}{l}H_{1}=K^{-1} d\left(\frac{1}{\phi^{\prime}}\right) \\ H_{2}=K^{2} d\left(\frac{1}{\phi^{\prime}}\right)\end{array}\right.$

It could then be rewritten as follows:

$\gamma G+v H_{1} M_{1}+v H_{2} M_{2}-M=0$

Thus:

$G=\gamma^{-1}\left(M-v H_{1} M_{1}-v H_{2} M_{2}\right)$

$\gamma_{1}=d\left(\phi^{\prime}\right)^{2} K^{2}+v\left[d\left(\phi^{\prime \prime}\right)+d(b) d\left(\phi^{\prime}\right)\right] K^{(k)}+v^{2} d(c)$, then $\gamma=\frac{1}{v^{2}} \gamma_{1}$ can be obtained. Then suppose $\left\|\gamma_{1}^{-1}\right\| \leq c_{1},\left\|H_{1}\right\| \leq c_{2}$, $\left\|H_{2}\right\| \leq c_{3}$, and $m_{1}(t, g(t)), m_{2}(t, g(t)), s(t, g(t))$ satisfies the Lipschitz condition:

$\left\{\begin{array}{l}\left\|m_{1}\left(t, g_{1}\right)-m_{1}\left(t, g_{2}\right)\right\| \leq\left\|g_{1}-g_{2}\right\| \\ \left\|m_{2}\left(t, g_{1}\right)-m_{2}\left(t, g_{2}\right)\right\| \leq\left\|g_{1}-g_{2}\right\| \\ \left\|s\left(t, g_{1}\right)-s\left(t, g_{2}\right)\right\| \leq\left\|g_{1}-g_{2}\right\|\end{array}\right.$

If the system of integral and differential equations can be solved Volterra, it should be satisfied that:

$\left|v^{2} c_{1}\right|\left(\left\|g_{1}-g_{2}\right\|+v\left|c_{2}\left\|g_{1}-g_{2}\right\|\right|+v\left|c_{3}\left\|g_{1}-g_{2}\right\|\right|\right) \leq 1$

Thus:

$v^{2}\left|c_{1}\left\|g_{1}-g_{2}\right\|\right|+v^{3}\left|c_{1} c_{2}\right|+v^{3}\left|c_{1} c_{3}\right| \leq 1$

The first choice in the actual calculation needs to select the appropriate $n$ value, so that the $v$ value calculated by the $v=\sqrt{\frac{\pi l}{n}}$, at the same time meet all the above conditions, at this time the Volterra integral differential equation group outer boundary value problem preprocessing is completed, can carry on the next calculation. However, the above is only for the external value problem of homogeneous boundary conditions, 
and also for the external value problem of non-homogeneous boundary conditions.

D. Treatment of Outside Value Problems of Nonhomogeneous Boundary Conditions

When the boundary condition of the outer boundary value problem of the Volterra integral differential equation group is nonhomogeneous, that is, $g(b)=\alpha \neq 0, g(c)=\beta \neq 0$, it is necessary to convert its nonhomogeneous boundary condition to homogeneous condition $g(b)=\alpha=0, g(c)=\beta=0$ at this time, using the following conversion method:

$f(x)=g(x)-\frac{c-x}{c-b} \alpha-\frac{x-b}{c-b} \beta$

Translating the issue into:

$f^{\prime \prime}(x)+b(x) f^{\prime}(x)+c(x) f(x)$

$+\int_{\alpha}^{x} m_{1}\left(t, f(t)+\frac{c-t}{c-b} \alpha+\frac{t-b}{c-b} \beta\right) d t$

$+\int_{\alpha}^{c} m_{2}\left(t, f(t)+\frac{c-t}{c-b} \alpha+\frac{t-b}{c-b} \beta\right) d t$

$=\hat{s}(x, f(x))$

where $x, t$ belong to the interval $[b, c]$ and $f(b)=0$,

$f(c)=0$. By sorting out (51), we can obtain:

$\hat{s}(x, f(x))=$

$s\left(x, f(x)+\frac{c-x}{c-b} \alpha+\frac{t-b}{c-b} \beta\right)-b(x)\left(\frac{\beta-\alpha}{c-b}\right)-c(x)\left(\frac{c-x}{c-b} \alpha+\frac{t-b}{c-b} \beta\right)$

By the above process, the problem Volterra the outer boundary value of the integral and differential equations is preprocessed, and the final calculation can be carried out according to the actual demand.

\section{EXPERIMENTAL COMPARISON}

Different pretreatment methods are used to deal with the same equation, and the external boundary value problem of Integro differential equations is obtained. The experimental environment is Windows 7, with $2 \mathrm{G}$ memory, 2.0 GHz CPU and $10 \mathrm{~g}$ free disk space. The Integro differential equation is generated by using the mathematical software MATLAB, and the true solution of the equation is obtained by using the internal function of the software.

Do a second order differential equation with Mathematica software, as shown in Fig. 4.

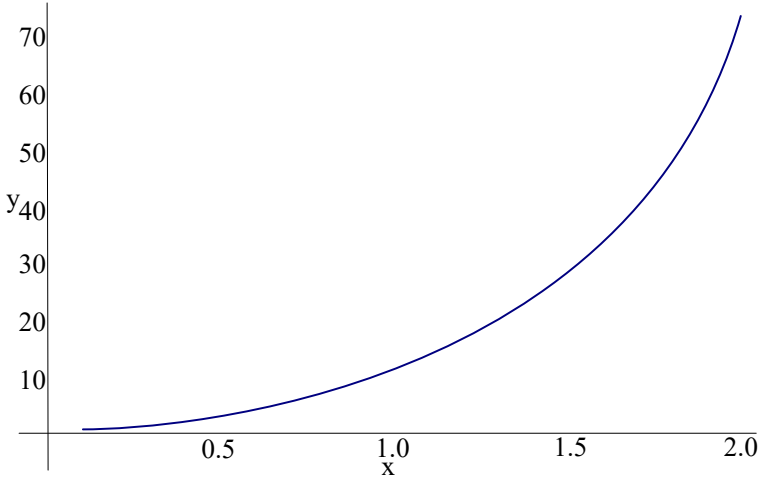

Fig. 4 integral differential equations

As can be seen from Fig. 4, X and $\mathrm{y}$ of the second-order differential equation show a positive correlation. In the course of the experiment, the series of equations are set to 16 and 32 , and two experiments are carried out to ensure the validity of the experimental comparison.

Under the above experimental conditions, the preprocessing method of the outer boundary value problem of the Volterra integral differential equations based on the spectral method is used as the first group of experiments, and the preprocessing method of the outer boundary value problem of the Volterra integral differential equations is designed as the second group of experiments. Tripartite software outputs experimental results. The experimental results of the external boundary value preprocessing method of the differential equations based on the spectral method are as shown in Fig. 5:

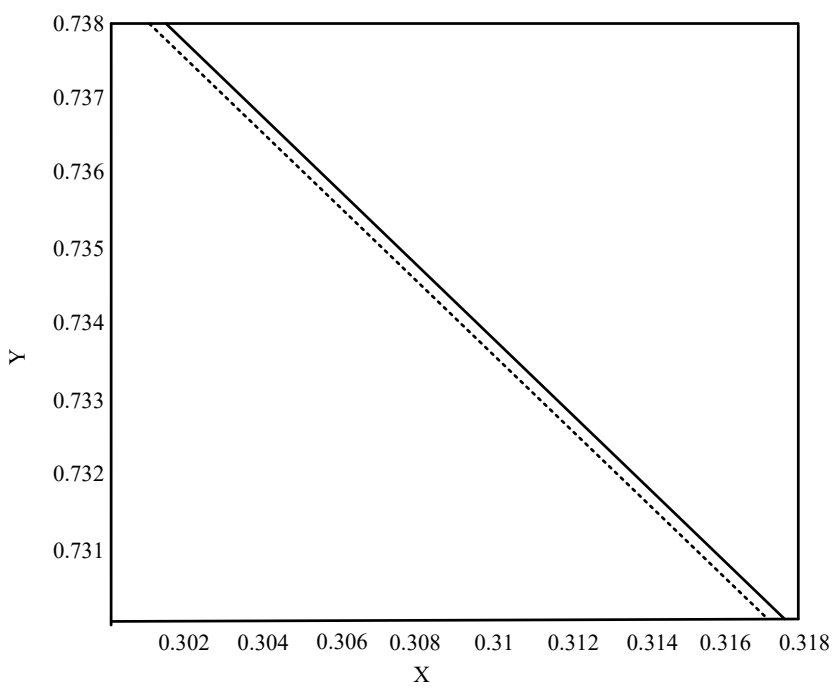

(a) experimental results with series 16 


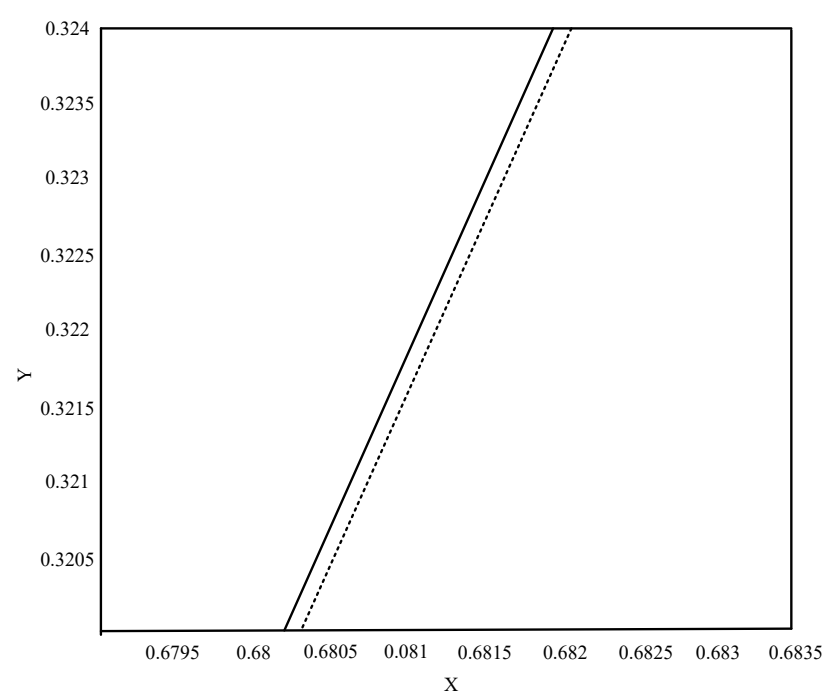

(b) experimental results with series 32

Fig. 5 experimental results of pre-treatment method of spectral method

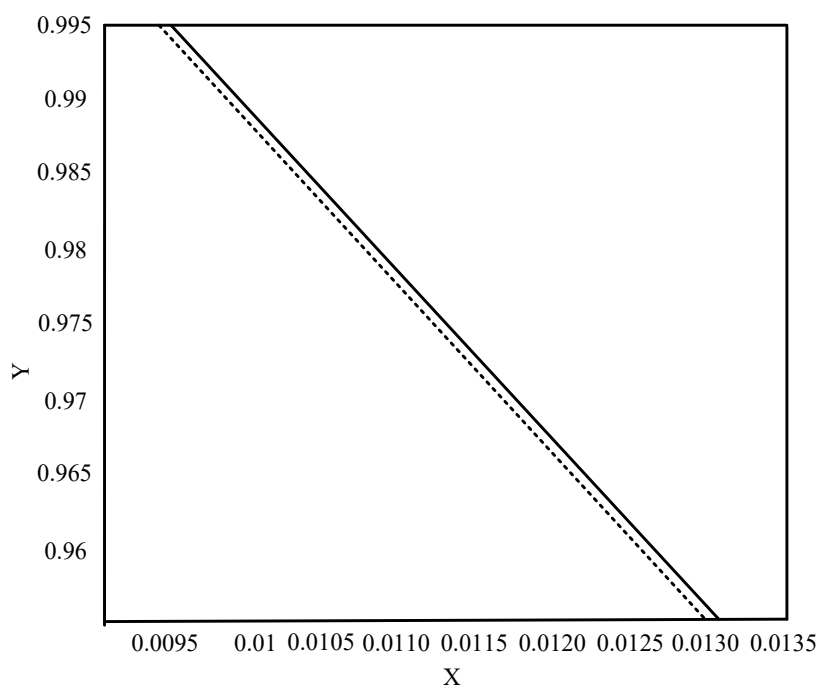

(a) experimental results with series 16

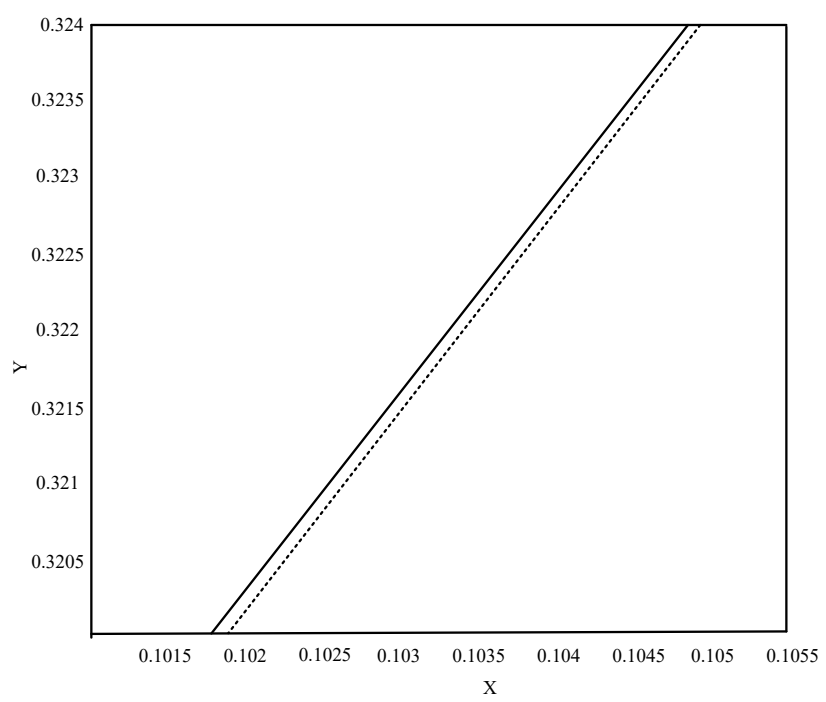

(b) experimental results with series 32
Fig. 6 experimental results of the designed pretreatment method

Observe the experimental results, the experimental results of the external value preprocessing method of differential equations based on spectral method are shown in Fig. 5. After calculation, the error ratio is 2.3911 at the series of 16 , and 6.8042 at the series of 32. Experimental results of the designed pretreatment method Volterra the problem of outer boundary value of the integral differential equation group is shown in Fig. 6 . the error ratio is 1.005 when the series is 16 , and 1.9036 when the series is 32. Compared with the results of the two groups, the spectral method pretreatment method is twice as designed when the series is 16 , and the error ratio is three times as designed when the series is 32 . To sum up, with the increase of series, the error rate of spectral preprocessing method increases, which is much higher than that of Volterra Integro differential equation external boundary value problem preprocessing method. The results show that the Volterra method is better than the spectral method.

\section{CONCLUSION}

The method designed to Volterra the problem of the outer boundary value of the integral differential equation group, by transforming the differential equation into the Volterra integral equation and using the Taylor to expand the equations, is convenient to preprocess the problem of the outer boundary value from the homogeneous boundary condition and the nonhomogeneous boundary condition, which is of great significance to the further study of the outer boundary value problem of the Volterra integral differential equation group. However, in the research process of this paper, no more experiments have been carried out on the external boundary value problem of Volterra integral equations, and the effect of the design method in other aspects cannot be determined. Further research is needed in the next research process.

\section{References}

[1] N. V. Denisova, "Instability degree and singular subspaces of integral isotropic cones of linear systems of differential equations," Doklady Mathematics, vol. 97, no. 1, pp. 35-37, 2018.

[2] R. N. Lee and A. A. Pomeransky, "Differential equations, recurrence relations, and quadratic constraints for L -loop two-point massive tadpoles and propagators," Journal of High Energy Physics, no. 8, pp. 1-27, 2019.

[3] A. Abdeljawad, M. Cappiello, and J. Toft, "Pseudo-differential calculus in anisotropic Gelfand-Shilov setting," Integral Equations and Operator Theory, vol. 91, no. 3, pp. 1-33, 2019.

[4] S. L. Ye, "Products of Volterra-type operators and composition operators from analytic morrey spaces into zygmund spaces," WSEAS Transactions on Mathematics, no. 18 , pp. 378-388, 2019.

[5] G. Mehdiyeva, V. Ibrahimov, and M. Imanova, "On the construction of the advanced hybrid methods and application to solving Volterra integral equation," WSEAS 
Transactions on Systems and Control, no. 14, pp. 183-189, 2019.

[6] I. G. Burova, "Application local polynomial and non-polynomial splines of the third order of approximation for the construction of the numerical solution of the Volterra integral equation of the second kind," WSEAS Transactions on Mathematics, no. 20, pp. 9-23, 2021.

[7] Y. M. Chen and X. J. Zhang, "Numerical solution of fractional nonlinear sine Gordon equation by shifted Chebyshev polynomial," Journal of Liaoning University of Engineering and Technology: Natural Science Edition, vol. 38, no. 1, pp. 92-96, 2019.

[8] Y. Kang and X. D. Zhang, "Approximate analytical and numerical solutions of fractional telegraph equation," Journal of Beijing University of Technology and Technology: Natural Science Edition, vol. 26, no. 6, pp. 68-72, 2008.

[9] W. Zhang, "Solution of fractional Volterra integral equations by collocation method based on improved hat function," Journal of Chengdu Normal University, vol. 323, no. 1, pp. 120-125, 2020.

[10] J. Y. Wei, S. Y. Wang, and Y. J. Li, "Existence of positive solutions for boundary value problems of a class of semi positive second-order ordinary differential equations," Journal of Shandong University (Science Edition), vol. 54, no. 10, pp. 11-16, 2019.

[11] J. Q. Gao and Y. C. Yang, "Proof method of asymptotic behavior of solution of a second order ordinary differential equation," Henan Science, vol. 234, no. 5, pp. 7-11, 2018.

[12] Q. Bao, S. Y. Fan, and X. C. Huang, "Bilinear interpolation deflection footprint correction technology based on Taylor's second-order expansion," Journal of People's Public Security University of China (Science and Technology), vol. 101, no. 3, pp. 11-16, 2019.

[13] S. K. Guo and W. Tang, "Bessel function and sinc function in optics," Journal of Xuzhou Normal University, vol. 18, no. 1, pp. 30-32, 2000.

[14] T. Ibukiyama, "Generic differential operators on Siegel modular forms and special polynomials," Selecta Mathematica, vol. 26, no. 5, pp. 230-250, 2020.

[15] Y. Tang, D. F. Zhao, Z. B. Huang, and Z. T. Dai, "High performance row task hash GPU general sparse matrix matrix multiplication," Journal of Beijing University of Posts and Telecommunications, vol. 42, no. 3, pp. 106-113, 2019.

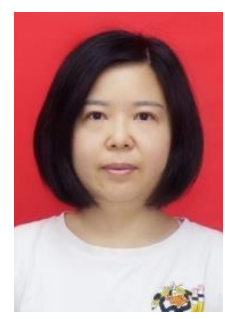

Xiaojuan Chen, female, was born in December 1986. Her professional title is lecturer. She received a bachelor's degree in horticulture from Jilin University in 2010. She received a PH.D. in horticulture and vegetable science from Zhejiang University in 2015. She is now working in Chongqing Vocational College of Transportation. Her field of study includes ideological and political education. She has published four academic papers. She is also involved in three research projects.

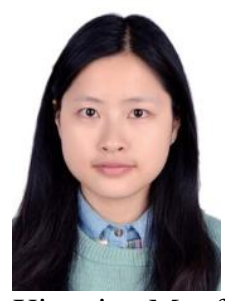

Xiaoxiao Ma, female, was born in May 1990. Her professional title is lecturer. She received her bachelor's degree in agricultural resources and environment from Huazhong Agricultural University in 2012. She received her master's degree in plant nutrition from Zhejiang University in 2015. She now works at Chongqing Vocational College of Transportation. Her areas of research include applied mathematics. She has published five academic papers. She is also involved in three research projects.

\section{Author Contributions:}

Xiaojuan Chen studied the external boundary value problem of Volterra Integro differential equation. Xiaoxiao Ma did experiments and comparisons.

\section{Creative Commons Attribution License 4.0 (Attribution 4.0 International, CC BY 4.0)}

This article is published under the terms of the Creative Commons Attribution License 4.0 https://creativecommons.org/licenses/by/4.0/deed.en_US 